\title{
Research on Steering Stability Control Strategy of Four-wheel Independent Electric Drive Special Vehicles
}

\author{
LIAO Zili ${ }^{1}$, SHU Xin ${ }^{1,2, *}$, CAI Lichun ${ }^{1}$, ZHANG Linyun ${ }^{1,3}$ \\ ${ }^{1}$ Army Academy of Armored Force, Beijing 100072, China; \\ 2.Troops 71345 of PLA, Zibo 255000, China; \\ 3. Troops 61623 of PLA, Beijing 100080, China
}

\begin{abstract}
In order to solve the steering stability problem of a special four-wheel independent electric vehicle, a dynamic model of the vehicle was established to analyze the cause of vehicle instability. A steering stability controller was designed, which included the upper yaw moment controller and the lower driving force distribution controller. Based on the sliding mode control algorithm, the yaw moment controller determines the yaw moment required while the vehicle is moving by controlling the yaw rate and sideslip angle. Based on the safety distribution method, the driving force distribution controller selects the sum of tire utilization rate as the optimization function to realize the optimal distribution of yaw moment. Software of MATLAB/Simulink and CarSim were used to build a co-simulation platform, and the designed steering stability controller was analyzed and verified. The simulation results show that the steering stability controller is helpful to improve the steering ability and handling stability of the vehicle in the extreme working conditions.
\end{abstract}

\section{Introduction}

With the development of motor and control technology, electric vehicles, with their characteristics of flexible drive, high efficiency and good controllability, have been highly regarded by major military countries in the world, and have been applied more and more widely in the military field. The four-wheel independent electric drive special vehicle adopts the distributed drive mode, each motor is independent and controllable, compared with the traditional internal combustion engine vehicle, it eliminates the clutch, transmission, transmission shaft and other transmission devices, simplifies the chassis structure, reduces the weight of the car body, so it has better flexibility and maneuverability ${ }^{[1]}$.

However, with the increase of freedom of control and the complicated driving conditions, the stability control of special four-wheel independent electric drive vehicle is required to be higher. In some extreme working conditions, such as emergency lane change or continuous avoidance of obstacles, the driver may be unable to make timely adjustments, and the vehicle is likely to exceed its stability margin, resulting in the danger of loss of control ${ }^{[2]}$. Therefore, a steering stability controller is designed in this paper to give full play to the advantages of electrically driven vehicles and effectively improve the steering stability of vehicles.

\section{Structure of four-wheel independent electric drive special vehicle}

The design requirements of a four-wheel independent electrically driven special vehicle are as follows: the maximum speed is $122 \mathrm{~km} / \mathrm{h}$, the average off-road speed is $35 \mathrm{~km} / \mathrm{h}$, the maximum angel of climb is $60 \%$, and the minimum turning radius is $1.2 \mathrm{~m}$. According to the requirements of its design indicators, considering comprehensively the matching relationship among power, transmission, steering and braking system, the electric transmission system scheme of "engine-generator set and power battery hybrid integration" and "hub motor fourwheel independent drive" is adopted. The vehicle system structure is shown in Figure $1^{[3]}$. 


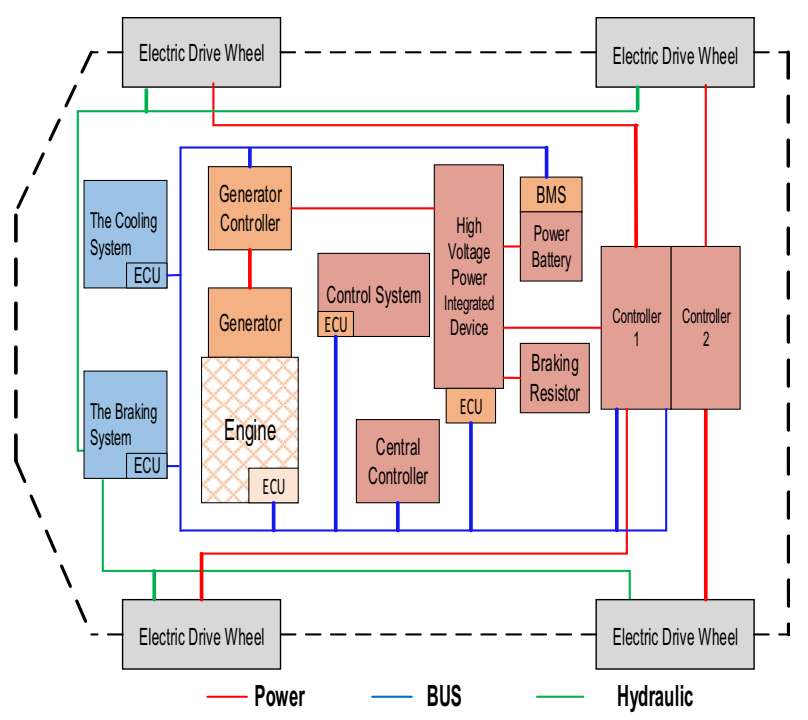

Fig. 1. System structure diagram of four -wheel independent electric drive vehicle

\section{Vehicle dynamics model}

\subsection{Vehicle 7-DOF model}

Vehicle is a multi-degree-of-freedom and strongly nonlinear system, so it is necessary to simplify the model of vehicle. The yaw moment distribution of the vehicle is mainly affected by the lateral force and longitudinal force of the tire, and the roll, pitch and vertical movement of the vehicle are ignored. Therefore, a seven-degree-of-freedom model of the vehicle is established, as shown in Figure 2 below ${ }^{[4]}$

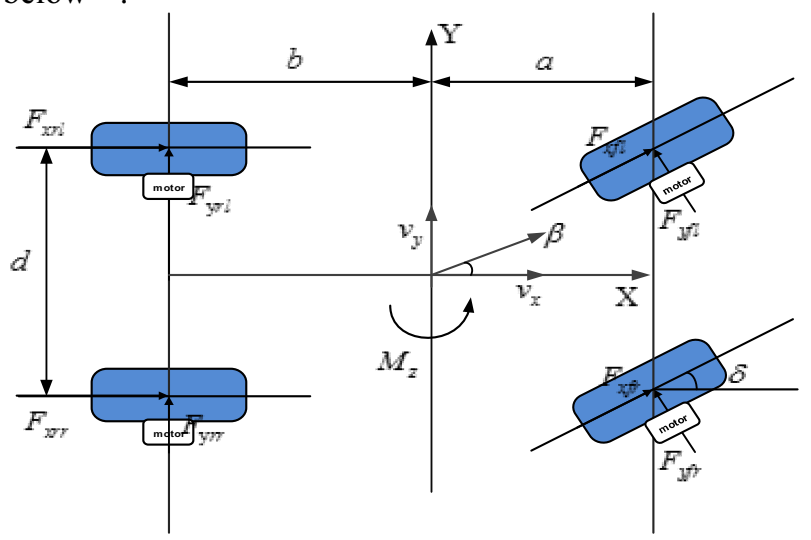

Fig. 2. Vehicle 7-DOF model

The total longitudinal force of the four tires satisfies the following equation:

$$
F_{d}=F_{x f l} \cos \delta+F_{x f r} \cos \delta+F_{x r l}+F_{x r r}
$$

The total yaw moment of four tires satisfies the following equation:

$$
M_{z}=\left(F_{x f l} \cos \delta-F_{x p r} \cos \delta+F_{x x l}-F_{x r}\right) \frac{d}{2}+a F_{x p l} \sin \delta+a F_{x p r} \sin \delta
$$

The lateral load transfer can be ignored and only the longitudinal load transfer can be considered when the vehicle is turning. Vertical load formula of each tire is as follows:

$$
\begin{aligned}
& F_{z f l}=m g \frac{b}{2 l}-m \dot{v}_{x} \frac{h_{g}}{2 l}-m \dot{v}_{y} \frac{h_{g}}{d} \cdot \frac{b}{l} \\
& F_{z f r}=m g \frac{b}{2 l}-m \dot{v}_{x} \frac{h_{g}}{2 l}+m \dot{v}_{y} \frac{h_{g}}{d} \cdot \frac{b}{l} \\
& F_{z r l}=m g \frac{a}{2 l}+m \dot{v}_{x} \frac{h_{g}}{2 l}-m \dot{v}_{y} \frac{h_{g}}{d} \cdot \frac{a}{l} \\
& F_{z r r}=m g \frac{a}{2 l}+m \dot{v}_{x} \frac{h_{g}}{2 l}+m \dot{v}_{y} \frac{h_{g}}{d} \cdot \frac{a}{l}
\end{aligned}
$$

The formula of side angle of each tire is as follows:

$$
\begin{aligned}
& \alpha_{f l}=\delta-\arctan \left(\frac{v_{y}+a \omega}{v_{x}-\omega d / 2}\right) \\
& \alpha_{f r}=\delta-\arctan \left(\frac{v_{y}+a \omega}{v_{x}+\omega d / 2}\right) \\
& \alpha_{r l}=-\arctan \left(\frac{v_{y}-a \omega}{v_{x}-\omega d / 2}\right) \\
& \alpha_{r r}=-\arctan \left(\frac{v_{y}-a \omega}{v_{x}+\omega d / 2}\right)
\end{aligned}
$$

Among them, $F_{x f l} 、 F_{x f r} 、 F_{x r l} 、 F_{x r r}$ are respectively the longitudinal forces on the left front, right front, left rear and right rear wheels, $F_{z f l} 、 F_{z f r}, F_{z r l} 、 F_{z r r}$ are respectively vertical load of left front, right front, left rear and right rear wheels, $\alpha_{f l}, \alpha_{f r}, \alpha_{r l}, \alpha_{r r}$ are respectively the sideslip angles of left front, right front, left rear and right rear wheels, $m$ is quality, $v_{x}$ is vertical speed, $v_{y}$ is lateral speed, $\omega$ is the yaw rate, $\delta$ is the front wheel angle, $a$ is the distance from the front axis to the center of mass, $b$ is the distance from the rear axis to the center of mass, $l=a+b$ is wheelbase, $d$ is wheel track, $h_{g}$ is height of the center of mass.

\subsection{Vehicle linear 2-DOF model}

In order to analyze the basic state of vehicle steering stability, a linear two-degree-of-freedom model was selected considering the ideal input-output relationship between the front wheel steering angle and vehicle yaw motion, as shown in Figure $3^{[5]}$.

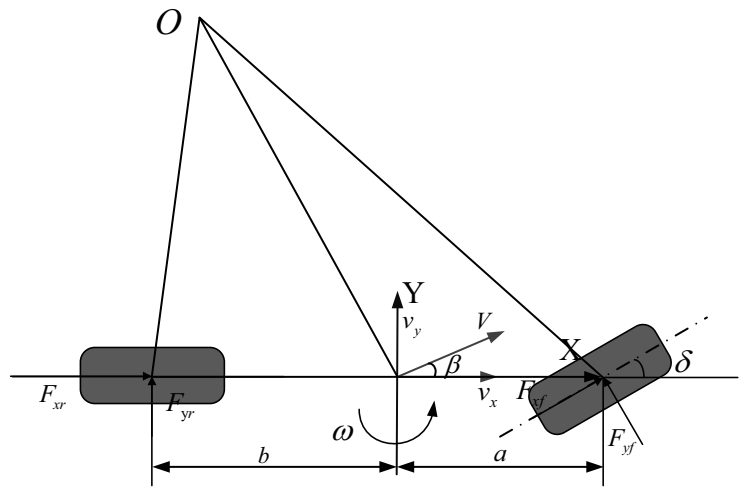

Fig. 3. Vehicle linear 2-DOF model

The dynamic equation of a two-degree-of-freedom vehicle is: 


$$
\begin{gathered}
m a_{y}=F_{y f}+F_{y r} \\
I_{z} \dot{\omega}=F_{y f} a+F_{y r} b
\end{gathered}
$$

Among them, the lateral force of the wheel $F_{s} 、 F_{r r}$ can be expressed as follows:

$$
F_{v f}=C_{f} \cdot \alpha_{f}, F_{v r}=C_{r} \cdot \alpha_{r}
$$

In the math above, $C, C$, are respectively the lateral stiffness of the front and rear wheels of the vehicle, and $a_{5} 、 \alpha_{r}$ are respectively the side angle of the front and rear wheels.

The relationship between vehicle lateral deviation angle and sideslip angle is as follows:

$$
\begin{gathered}
a_{f}=\beta+\frac{a \omega}{v_{x}}-\delta \\
a_{r}=\beta-\frac{b \omega}{v_{x}}
\end{gathered}
$$

According to the above equation, the kinematics equation of the vehicle's 2-DOF model can be deduced as follows:

$$
\begin{aligned}
& m\left(\dot{v}_{y}+v_{x} \omega\right)=\left(C_{f}+C_{r}\right) \beta+\frac{1}{v_{y}}\left(a C_{f}-b C_{r}\right) \omega-C_{f} c \\
& I_{z} \dot{\omega}=\left(a C_{f}-b C_{r}\right) \beta+\frac{1}{v}\left(a^{2} C_{f}-b^{2} C_{r}\right) \omega-
\end{aligned}
$$

\subsection{Vehicle tire model}

The dynamic response of vehicle steering is mainly affected by tire characteristics. In order to accurately represent the nonlinear characteristics of tires and meet the real-time requirements of simulation tests, the Dugoff tire model is selected to calculate the longitudinal and lateral forces of tires, and the expressions are as follows ${ }^{[6]}$ :

$$
\begin{gathered}
F_{x i}=f(\lambda) \cdot C_{x i} \cdot s_{i} \\
F_{y i}=f(\lambda) \cdot C_{y i} \cdot \alpha_{i}
\end{gathered}
$$

Among them,

$$
\begin{gathered}
\lambda=\frac{\mu_{\max } F_{z i}}{\sqrt{\left(C_{x i} \cdot s_{i}\right)^{2}+\left(C_{y i} \cdot \alpha_{i}\right)^{2}}} \\
f(\lambda)= \begin{cases}(2-\lambda) \lambda, & \lambda<1 \\
1, & \lambda>1\end{cases}
\end{gathered}
$$

In the above formula, $c_{n}, c_{n}$ are the longitudinal and lateral stiffness of the tire, ${ }^{\alpha}$ is the side-slip angle of the tire, $F_{z i}$ is the vertical load of the tire, $\mu_{\max }$ is the peak adhesion coefficient of the road surface, and $S_{i}$ is the tire slip rate.

\subsection{Permanent magnet synchronous motor model}

Permanent magnet synchronous motor has the advantages of small volume, light weight, high power density and good reliability, so the selection of permanent magnet synchronous motor model can meet the off-road mobility and stability requirements of special electric vehicles.
With the rapid development of motor control technology, the motor can basically output according to the given torque at present. Therefore, the torque model of the motor is established by using the external characteristic curve of the motor in this paper, as shown in Figure 4.

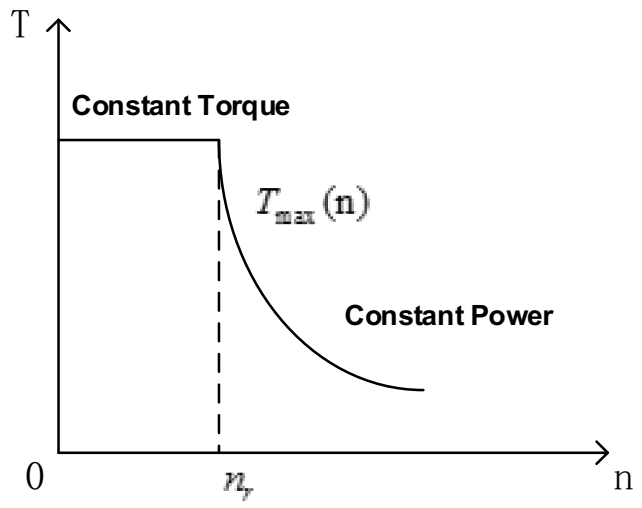

Fig.4.Motor external characteristic curve

It can be simplified into a first-order dynamic system model, as follows ${ }^{[7]}$ :

$$
\begin{aligned}
& T_{e}=\frac{1}{\tau S+1} T_{e_{-} \text {ref }} \\
& T_{e_{-} \text {ref }}=\eta T_{\text {max }}(n)
\end{aligned}
$$

$\eta$ is the motor efficiency, $T_{e_{-} r e f}$ and $T_{e}$ are the given value and actual value of the motor torque, and $T_{\text {mix }}(n)$ is the maximum torque of the motor at the current speed. In the actual driving process of the vehicle, when the speed (motor speed) is greater than the turning speed $n_{r}$, the vehicle is controlled by constant power. When the motor speed is less than the turning speed $n_{r}$, it is constant torque control.

\section{Analysis of vehicle instability reasons}

When the speed of the vehicle is fast, the external interference or the situation of the road surface is more complex, it is possible to appear the situation of vehicle instability. The heading angle of the vehicle can represent the movement direction of the vehicle, and its relationship with the yaw rate and sideslip angle is as follows ${ }^{[8]}$ :

$$
\Psi=\beta+\int \omega \cdot d t
$$

It can be seen from Equation (24) that when the sideslip angle is small, the heading angle is mainly determined by yaw angle. Therefore, the steering characteristics of the vehicle can be expressed by yaw rate. When the sideslip angle of the vehicle is large, both of them represent the steering characteristics of the vehicle.

\section{Design of steering stability controller for special vehicles}

The vehicle steering stability controller is divided into two layers, shown in Figure 5. The upper layer is the sliding mode controller which determines the yaw torque required by vehicle driving by controlling the yaw rate and sideslip 
angle, and the lower layer is the driving force distribution controller which distributes the yaw torque to the four wheels according to the optimal method.

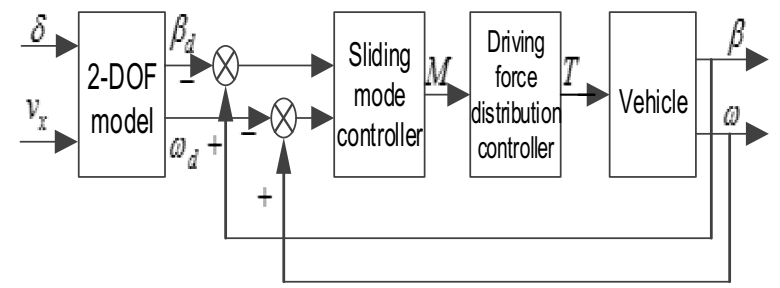

Fig.5. Combined sliding mode controller structure of yaw rate and sideslip angle

\subsection{Design of yaw moment controller based on sliding mode control}

Sliding mode variable structure control is a nonlinear control algorithm, which can make the system state move according to the sliding mode surface by switching control quantity ${ }^{[9]}$. This method has the advantages of fast dynamic response, simple control, strong robustness and good reliability. According to the 2-DOF reference model, the ideal value of the yaw rate is:

$$
\omega_{d}=\min \left\{\begin{array}{l}
\frac{v_{x}}{l\left(1+K v_{x}^{2}\right)} \delta \\
\left|\frac{\mu g}{v_{x}}\right| \cdot \operatorname{sgn}(\delta)
\end{array}\right.
$$

The ideal value of the sideslip angle is:

$$
\beta_{d}=\min \left\{\begin{array}{l}
\frac{b-m a v_{x}{ }^{2} /\left(C_{r} l\right)}{l\left(1+K v_{x}{ }^{2}\right)} \delta \\
\mu g\left(\frac{b}{v_{x}{ }^{2}}+\frac{m a}{C_{r}}\right)
\end{array}\right.
$$

Among them, $K=\frac{m}{l^{2}}\left(\frac{a}{C_{r}}-\frac{b}{C_{f}}\right)$.

After the yaw moment is applied, the 2-DOF model of the vehicle is:

$$
\left\{\begin{array}{l}
\dot{\beta}=\frac{C_{f}+C_{r}}{m v_{x}} \beta+\left(\frac{a C_{r}-b C_{r}}{m v_{x}^{2}}-1\right) \omega-\frac{C_{f}}{m v_{x}} \delta \\
\dot{\omega}=\frac{a C_{f}-b C_{r}}{I_{z}} \beta+\frac{a^{2} C_{f}+b^{2} C_{r}}{I_{z} v_{x}} \omega-\frac{a C_{f}}{I_{z}} \delta+\frac{\Delta M}{I_{z}}
\end{array}\right.
$$

In the above formula, $\Delta M$ is the additional yaw moment.

Generally, the vehicle is expected to have a faster response to the change of the yaw rate. Therefore, the tracking error and its change rate of the yaw rate is selected as follows:

$$
\begin{aligned}
& e_{\omega}=\omega-\omega_{d} \\
& \dot{e}_{\omega}=\dot{\omega}-\dot{\omega}_{d}
\end{aligned}
$$

The tracking error of the sideslip angle is:

$$
e_{\beta}=\beta-\beta_{d}
$$

The sliding surface is defined as:

$$
\begin{aligned}
& s=c_{1}\left(c_{2} e_{\omega}+\dot{e}_{\omega}\right)+\left(1-c_{1}\right) e_{\beta} \\
& \dot{s}=c_{1}\left(c_{2} \dot{e}_{\omega}+\ddot{e}_{\omega}\right)+\left(1-c_{1}\right) \dot{e}_{\beta} \\
& =c_{1} c_{2} \dot{e}_{\omega}+c_{1}\left(\ddot{\omega}-\ddot{\omega}_{d}\right)+\left(1-c_{1}\right) \dot{e}_{\beta}
\end{aligned}
$$

In the above formula, $c_{1}$ is the weight coefficient of yaw rate, $0<c_{1}<1, s$ is the sliding mode variable. While $s \rightarrow 0, \dot{s} \rightarrow 0, \quad e_{\omega} \rightarrow 0, \quad \dot{e}_{\omega} \rightarrow 0, \quad e_{\beta} \rightarrow 0$.

According to Equations (27) and (32), the additional yaw moment of sliding mode control can be determined as:

$$
\begin{aligned}
& \Delta M=-I_{z}\left(c_{2} \dot{e}_{\omega}+\frac{1-c_{1}}{c_{1}} \dot{e}_{\beta} \frac{a C_{f}-b C_{r}}{I_{z}} \dot{\beta}+\right. \\
& \left.\frac{a^{2} C_{f}+b^{2} C_{r}}{I_{z} v_{x}} \dot{\omega}_{d}-\frac{a C_{f}}{I_{z}} \dot{\delta}_{-}-\ddot{\omega}_{d}+\varepsilon \operatorname{sgn}(s)\right)
\end{aligned}
$$

When the system parameters change or there is external disturbance, the control system may deviate from the sliding mode surface. Therefore, the above equation adds the switching function $\varepsilon \operatorname{sgn}(s)$ as the control law, so that the system can quickly return to the sliding mode surface. $\mathcal{E}$ is the approaching speed of the control law, is positive. $\operatorname{sgn}(s)$ is the sign function.

\subsection{Design of driving force distribution controller based on objective optimization}

In order to ensure the steering stability of special vehicles, the driving force distribution controller of the lower level takes the tire utilization rate as the optimization objective, and the yaw torque demand obtained from the upper level yaw torque controller is distributed to the four wheels through the allocation algorithm.

The tire utilization rate $\eta_{i}$ is the ratio of the road adhesion of a single wheel to the maximum adhesion the ground can provide. Equation (34) is as follows, which can be used to reflect the stability margin of the vehicle. The lower the tire utilization rate, the higher the stability margin of the automobile will be, the greater the fault tolerance rate and the better the stability.

$$
\eta_{i}=\frac{\sqrt{F_{x i}^{2}+F_{y i}^{2}}}{\mu_{i} F_{z i}}
$$

Among them, $F_{j i}$ is the lateral force of left front, right front, left rear and right rear wheels, and $\mu_{i}$ is the road adhesion coefficient. Four-wheel independent electrically driven special vehicles can only control the output torque of the motor. The motor torque acts on the longitudinal force of the tire through the reducer. Since the lateral force cannot be directly controlled, the objective function is selected as follows:

$$
\min J=\min \sum_{\mathrm{i}=1}^{4} \frac{F_{x i}{ }^{2}}{\left(\mu_{i} F_{z i}\right)^{2}}
$$

Based on the classical law of friction, the net force of friction acting on the tire and the ground does not exceed the product of the tire vertical load and the coefficient of friction on the ground. At the same time, considering that the force distributed to each tire can not exceed the maximum driving force that the hub motor can provide, the constraint conditions are: 


$$
\left\{\begin{array}{l}
0 \leq F_{x i} \cdot R \leq T_{\max } \\
0 \leq F_{x i} \leq \mu_{i} F_{z i}
\end{array}\right.
$$

$R$ is the effective radius of the tire.

When the objective function is a quadratic function and the constraint condition is linear, the quadratic programming method is generally used to solve the problem. Quadratic programming problem is a kind of nonlinear programming problem with special form, and its basic formula is ${ }^{[10]}$ :

$$
\begin{gathered}
\min f(x)=\frac{1}{2} x^{T} Q x+c^{T} x \\
\text { s.t. }\left\{\begin{array}{l}
A x \leq b \\
A_{e q} x=b_{\mathrm{eq}} \\
l b \leq x \leq u b
\end{array}\right.
\end{gathered}
$$

Among them, $Q_{\text {and }} A_{e q}$ are matrix, the rest are vectors.

Transforming Equations (35) and (36) into Equations (37) and (38), we can get:

$$
\begin{aligned}
& x=\left[F_{x f l}, F_{x f r}, F_{x r l}, F_{x r r}\right]^{\mathrm{T}} \\
& Q=\left[\begin{array}{cccc}
1 /\left(\mu_{\mathrm{n}} F_{z f}\right)^{2} & 0 & 0 & 0 \\
0 & 1 /\left(\mu_{f r} F_{z f r}\right)^{2} & 0 & 0 \\
0 & 0 & 1 /\left(\mu_{r l} F_{z r l}\right)^{2} & 0 \\
0 & 0 & 0 & 1 /\left(\mu_{r r} F_{z r r}\right)^{2}
\end{array}\right] \\
& A=[R, R, R, R]^{\mathrm{T}} \\
& b=\left[T_{\text {max }}, T_{\text {max }}, T_{\text {max }}, T_{\text {max }}\right]^{\mathrm{T}} \\
& A_{e q}=\left[\begin{array}{cccc}
1 & 1 & 1 & 1 \\
\cos \delta+a \sin \delta & -\cos \delta+a \sin \delta & 1 & -1
\end{array}\right] \\
& b_{e q}=\left[\begin{array}{c}
F_{d} \\
M_{Z}
\end{array}\right] \\
& l b=[0,0,0,0]^{\mathrm{T}} \\
& u b=\left[\mu_{\mathrm{fl}} F_{z f l}, \mu_{f r} F_{z f r}, \mu_{r l} F_{z r l}, \mu_{r r} F_{z r r}\right]^{\mathrm{T}}
\end{aligned}
$$

The above formula uses the Quadprog function of MATLAB to solve the optimal driving force acting on each tire, so as to obtain the torque size of each motor and realize the optimal distribution of yaw moment ${ }^{[8]}$.

\section{Simulation experiment and analysis}

MATLAB/Simulink and CarSim are used to build a cosimulation platform through the interface of importing and exporting variables between the software. The inputoutput relationship is shown in Figure 6 to verify the correctness and effectiveness of the control algorithm.

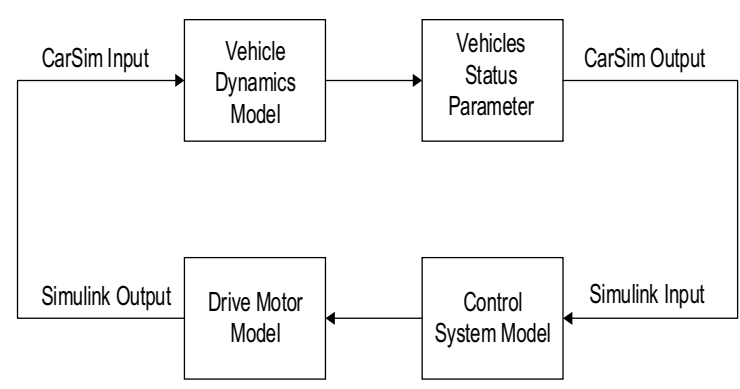

Fig.6. MATLAB/Simulink and CarSim co-simulation

The basic parameters and motor parameters of a fourwheel independent electrically driven special vehicle are shown in Table 1 and 2.

Table1 Vehicle parameters

\begin{tabular}{cc}
\hline \multicolumn{2}{c}{ Basic parameters of the vehicle } \\
\hline The quality of the vehicle & $6.4(\mathrm{t})$ \\
Drive form & $4 \times 4$ \\
Height of the center of mass & 1.617 \\
Wheel base & $3.3(\mathrm{~m})$ \\
Wheel track & $2.1(\mathrm{~m})$ \\
Tistance from front axis to center of mass & $1.617(\mathrm{~m})$ \\
Distance from rear axis to center of mass & $1.683(\mathrm{~m})$ \\
The moment of inertia & $11018\left(\mathrm{~kg} \cdot \mathrm{m}^{2}\right)$ \\
Radius of the tire & $0.52(\mathrm{~m})$ \\
\hline & \\
Table 2 Motor parameters & \\
\hline Hub motor basic parameters \\
\hline Rated power (kW) \\
Peak power $(\mathrm{kW})$ \\
Rated speed (r/min) \\
The highest speed (r/min) \\
The rated torque (Nm) \\
Peak torque (Nm)
\end{tabular}

According to the requirements of the steering transient response test, the simulation test conditions for: a given test speed is $80 \mathrm{~km} / \mathrm{h}$, the vehicle running in a straight line, 150 degree step input applied to the steering angle at $2 \mathrm{~s}$ (as shown in Figure 7(a)), then the vehicle turns according to the steering angle. The simulation was carried out on low adhesion road surface (adhesion coefficient was 0.3 ), the simulation results obtained are shown in Figure 7(b)-(f).

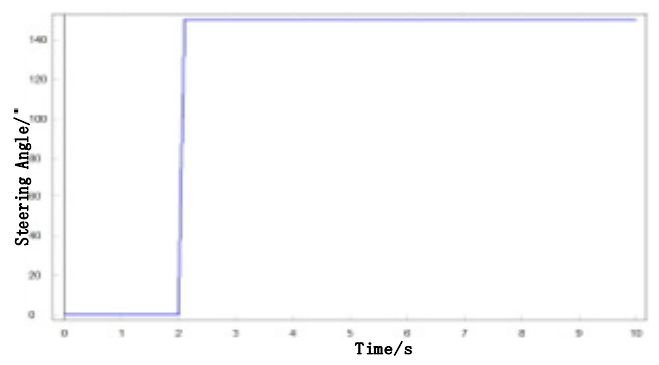

a. Steering wheel Angle input 


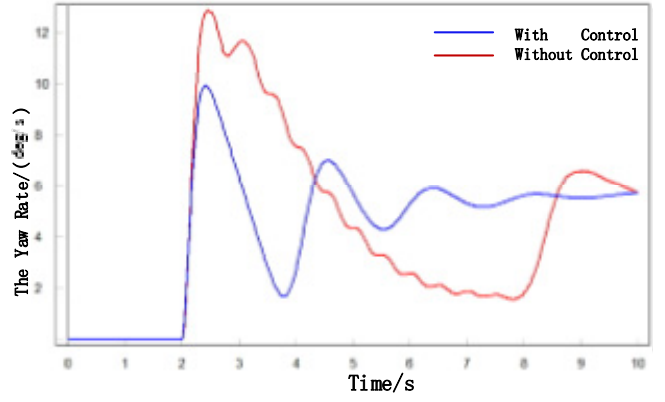

b. The curve of yaw rate with respect to time

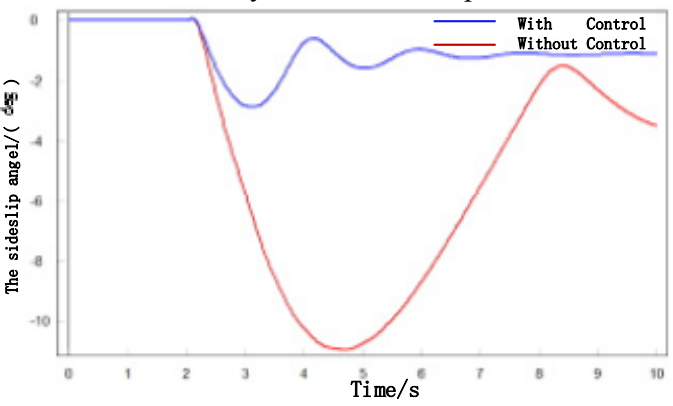

c. The curve of sideslip angle with respect to time

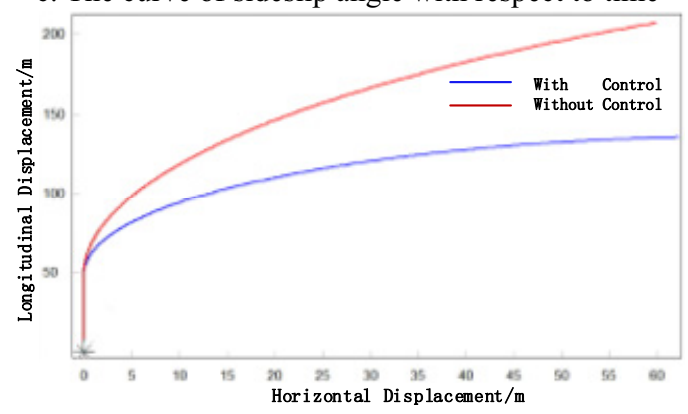

d. Movement track

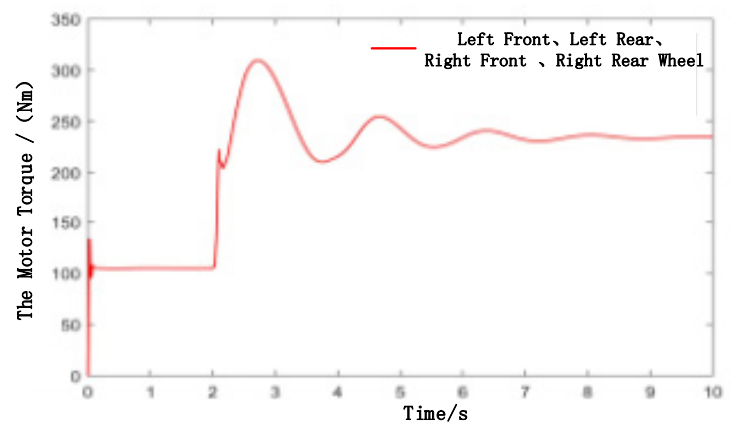

e. Motor torque response without control

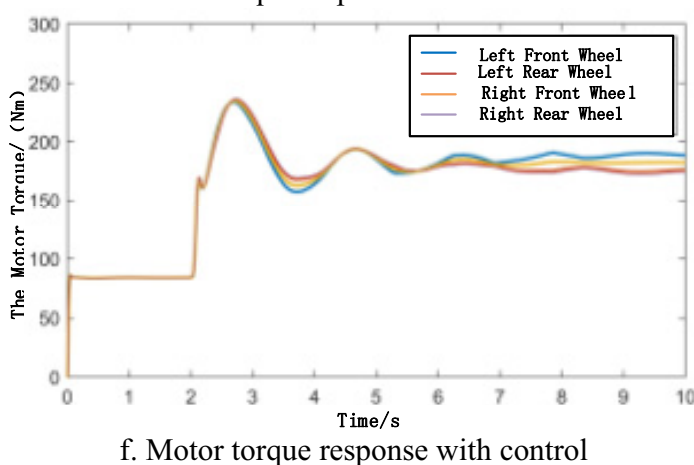

Fig.7. Transient steering response test of low adhesion road surface

When the vehicle on the low-adhesion road is steering at high speed, it can be seen from Figure (b) that the yaw rate of the vehicle is larger and fluctuates more sharply when the control is not applied. After the control is applied, the yaw rate of the vehicle decreases significantly and tends to be stable in the later stage, and the vehicle steering is more stable. As can be seen from Figure(c), when the control is not applied, the sideslip angle of the vehicle changes greatly and the vehicle is unstable. After the control is applied, the sideslip angle of the vehicle changes slightly and the vehicle has good stability. As can be seen from Figure (d), due to the insufficient steering yaw moment of the vehicle without control (that is to use equal torque distribution), the vehicle has a certain sideslipping, which leads to a larger steering radius and an offset of the vehicle's traveling trajectory. It can be seen from Figure (e) and (f), when no control is applied, the motor torque of the four wheels is the same due to the method of equal distribution. Due to the torque difference between the left and right wheels, the torque difference of the left wheel is greater than that of the right wheel when the vehicle is turning, so as to ensure that the vehicle can drive in accordance with the normal trajectory. At the same time, the motor torque is also significantly reduced, which reduces the vehicle's driving speed and ensures the stability of the vehicle steering.

\section{Conclusion}

In this paper, a steering stability controller, which includes yaw torque control and drive torque distribution, is designed for a special four-wheel independent electrically-driven vehicle. The effectiveness of the control algorithm is verified by the software of Matlab and CarSim co-simulation. The simulation results show that the proposed steering stability control strategy is effective to improve the steering stability of special four-wheel independent electric drive vehicles under extreme working conditions.

\section{References}

1. Ren Bingtao.Research on Torque Coordination and Optimal Control of Four Wheel Drive Electric Vehicle[D].Jilin University(2017).

2. Xu Linna.Research on Steering Stability Control for Four-wheel-Independent-Drive Electric Vehicle[D]. Dalian University of Technology(2019).

3. Cai Lichun.Research on Handling Stability Control of Electric Drive Vehicle with Four In-wheel Motors[D].Army Academy of Armored Forces(2019).

4. Wang Jie.Steering Stability Control of Four-Wheel Drive Vehicle with Hub Motors[D]. Beijing Institute of Technology(2015).

5. Masato Abe.Vehicle Handling Dynamics Theory and Application[M],China Machine Press (2016).

6. Rajesh Rajamani.Vehicle Dynamics and Control[M] , China Machine Press (2018).

7. Yang Guibing. Research on Motion Control Strategy for Electric Drive Wheeled Vehicle[D]. Academy of Armored Forces Engineering(2013). 
8. Lenzo B, Sorniotti A, Gruber P. A single input single output formulation for yaw rate and sideslip angle control via torque-vectoring[J],(2018).

9. Liu Jinkun. Sliding Mode Control Design and MATLAB Simulation [Third Edition]:The Basic Theory and Design Method[M], Tsinghua University Press(2015).

10. Harkegard O. Dynamic control allocation using constrained quadratic programming[J]. Journal of Guidance, Control, and Dynamics,27(6): 10281034(2004). 